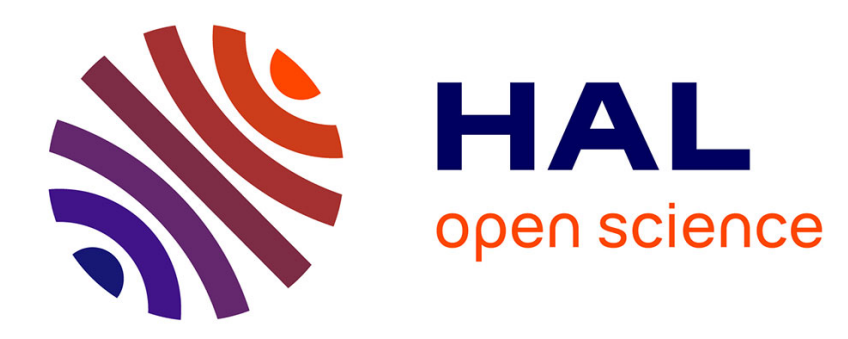

\title{
Crystal structure of $\alpha-\mathrm{Al} 2(\mathrm{CrO} 4) 2 \mathrm{Cr} 2 \mathrm{O} 7 \times 4 \mathrm{H} 2 \mathrm{O}$
}

Yannick Cudennec

\section{To cite this version:}

Yannick Cudennec. Crystal structure of $\alpha$-Al2(CrO4) $2 \mathrm{Cr} 2 \mathrm{O} 7 \times 4 \mathrm{H} 2 \mathrm{O}$. Journal of Inorganic and Nuclear Chemistry, 1977, 39 (9), pp.1711-1713. 10.1016/0022-1902(77)80136-X . hal-03025200

\section{HAL Id: hal-03025200 https://hal.science/hal-03025200}

Submitted on 26 Nov 2020

HAL is a multi-disciplinary open access archive for the deposit and dissemination of scientific research documents, whether they are published or not. The documents may come from teaching and research institutions in France or abroad, or from public or private research centers.
L'archive ouverte pluridisciplinaire HAL, est destinée au dépôt et à la diffusion de documents scientifiques de niveau recherche, publiés ou non, émanant des établissements d'enseignement et de recherche français ou étrangers, des laboratoires publics ou privés. 


\title{
Crystal structure of $\alpha-\mathrm{Al}_{2}\left(\mathrm{CrO}_{4}\right)_{2} \mathrm{Cr}_{2} \mathrm{O}_{7}, 4 \mathrm{H}_{2} \mathrm{O}$
}

\author{
YANNICK CUDENNEC \\ Laboratoire de Cristallochimie Minérale, Institut National des Sciences Appliquées 35031. Rennes Cedex \\ B.P.14A France.
}

(Received 15 January 1977)

\section{https://doi.org/10.1016/0022-1902(77)80136-X}

In a previous publication relating to the preparation of aluminum chromates, an aluminum chromate bichromate has been described [1]. The choice of this new formula was based solely, upon results of thermolysis and IR spectrophotometric studies. It was thus necessary to determine the crystal structure.

This determination has been carried out on the basis of an isomorphism with the homologous iron compound, the structure of which has been determined by Gérault et al.[2]

\section{CRYSTAL STRUCTURE DETERMINATION}

$\alpha-\mathrm{Al}_{2}\left(\mathrm{CrO}_{4}\right)_{2} \mathrm{Cr}_{2} \mathrm{O}_{7}, 4 \mathrm{H}_{2} \mathrm{O}$ occurs in the form of red needles. 1.aue photographs show it to be monoclinic. The systematic extinctions $h o l, l=2 n$ are consistent with the space group P2/c or Pc. The nonconventional centrosymetric space group $\mathrm{P} 2 / \mathrm{n}$ has been chosen as in the iron compound study. The unit-cell dimensions obtained and refined by least-squares methods from a powder pattern are:

--in the space group P2/c

$a=13,30(3) \AA ; b=10,48(2) \AA ; \quad c=14,32(3) \AA ; \beta=158,15(5)^{\circ} ; Z=2$

--in the space group $\mathrm{P} 2 / \mathrm{n}$

$a=5,329(6) \AA ; \quad b=10,48(2) \AA ; \quad c=13,30(3) \AA ; \beta=90,00(5)^{\circ} ; \quad Z=2$

During the whole intensity gathering process, the single crystal was placed in a dry nitrogen stream. MoK $\alpha$ radiation was used to minimize absorption effects which are weak for aluminum compounds, so that no correction for absorption was made. The reciprocal planes hkl $(1=0-6)$ have been collected on a Büerger apparatus. Integrated intensities were measured with a NONIUS microdensitometer and corrected solely, for LORENTZ and polarisation effects. 483 independant reflections were collected.

The form factors used are those of $\mathrm{Al}^{3+}, \mathrm{Cr}^{3+}, \mathrm{O}^{-1}$. They are computed from the Vand et al.[3] relation and coefficients taken from the Moore tables [4]. Computations have been carried out on a CII 10070 computer, using the refinement program SFLS5 [5].

The refinement of the atomic and isotropic thermal parameters was made from the iron chromate bichromate data. Four cycles of refinements reduced the residual $R=\Sigma\left|K_{0}-\right| F_{c}|| / \Sigma F_{0}$, to 0.082 .

Table 1 gives the final atomic and thermal parameters. Table 2 lists the interatomic bond distances 
and angles and their errors, calculated with the Busing et al. Program [6]. We give, below, the key which permits identification of each atom.

$\begin{array}{rrrr}\text { (i) } & x & y & z \\ \text { (ii) } & -x & -y & -z \\ \text { (iii) } 1 / 2+x & -y & 1 / 2+z \\ \text { (iv) } 1 / 2-x & y & 1 / 2-z \\ \text { (v) } & -1+x & y & z \\ \text { (vi) } 11-x & -y & -z \\ \text { (vii) } 3 / 2-x & y & 1 / 2-z \\ \text { (viii) } & x & -1+y & z \\ \text { (ix) } & 1-x & 1-y & -z\end{array}$

\section{DISCUSSION}

The results obtained confirm the original hypothesis for the compound formula. The solid phase belongs to a new family of compounds in which $\mathrm{CrO}_{4}{ }^{2-}$ and $\mathrm{Cr}_{2} \mathrm{O}_{7}{ }^{2-}$ groups coexist. It is isotypic with the iron compound. The hydration step is higher than the one deduced from chemical and thermogravimetric studies. This hygroscopic phase, as already mentioned. does not contain 3 but 4 water molecules. Observed analytical results are in close agreement with calculated-ones [1].

The average Al-O distance (1.89 $\AA)$ agrees well with that found in other compounds where the coordination polyhedron of aluminum is an octahedron. The average $\mathrm{Cr}-\mathrm{O}$ distance $(1.65 \AA)$ is similar to that observed in the iron chromate bichromate [2]. The shortest $\mathrm{Cr}-\mathrm{O}$ distance corresponds to an oxygen which does not belong to any other polyhedron; the longest one (1.76 $\AA$ ) belongs to the bridge $\mathrm{Cr}-\mathrm{O}-\mathrm{Cr}$. Bonds of similar length exist in alkaline bichromates.

The structure may be described as an imbrication of wavy sheets parallel with the plane (a, c). The only bond between the different sheets, are hydrogen bonds. The IR studies show relatively low frequencies for $v_{1}$ and $v_{3}$ of the water molecules [1], indicating the presence of hydrogen bonds. Table 2 gives the interatomic hydrogen bond distances and angles as defined by Gerault et al.[2].

\section{REFERENCES}

1. Y. Cudennec et A. Bonnin, J. Inorg. Nucl. Chem., 36, 273 (1974).

2. Y. Gerault et A. Bonnin, Bull. Soc. fr. Minéral. Cristallogr., 99, 197 (1976).

3. V. Vand, F. F. Eiland et R. Pepinski, Acta Cryst., 10, 303 (1957).

4. F. H. Moore, Acta Cryst., 16, 1169 (1963).

5. C. T. Prewitt, Fortran IV Full-Matrix Crvstallographic Least-Squares Program SFLS5 (1966).

6. W. R. Busing et H. R. Levy, Acta Cryst., 22, 457 (1967). 
Table 1. Final atomic and thermal parameters and standard deviations of $\alpha$ $\mathrm{Al}_{2}\left(\mathrm{CrO}_{4}\right)_{2} \mathrm{Cr}_{2} \mathrm{O}_{7} \cdot 4 \mathrm{H}_{2} \mathrm{O}$

\begin{tabular}{|c|c|c|c|c|}
\hline & $x$ & $y$ & $z$ & $B\left(\AA^{2}\right)$ \\
\hline $\mathrm{Al}_{1}$ & 0 & 0 & 0 & $0.21(11)$ \\
\hline $\mathrm{Al}_{2}$ & 0.25 & $0.3459(7)$ & 0.25 & $0.48(11)$ \\
\hline $\mathrm{Cr}_{1}$ & $0.5171(4)$ & $0.0953(3)$ & $0.1299(4)$ & $0.29(5)$ \\
\hline $\mathrm{Cr}_{2}$ & $0.5797(5)$ & $0.6029(3)$ & $0.1519(5)$ & $0.78(6)$ \\
\hline$O(1)$ & $0.8044(16)$ & $0.1131(9)$ & $0.0758(17)$ & $0.07(16)$ \\
\hline$O(2)$ & $0.2916(19)$ & $0.0874(11)$ & $0.046(2)$ & $0.52(18)$ \\
\hline $\mathrm{O}(3)$ & $0.514(2)$ & $-0.0315(13)$ & $0.192(2)$ & $1.6(3)$ \\
\hline $\mathrm{O}(4)$ & $0.468(2)$ & $0.2197(12)$ & $0.208(2)$ & $1.1(2)$ \\
\hline$O(5)$ & 0.75 & $0.6757(19)$ & 0.25 & $1.6(4)$ \\
\hline$O(6)$ & $0.367(3)$ & $0.7015(16)$ & $0.120(3)$ & $2.6(3)$ \\
\hline$O(7)$ & $0.466(3)$ & $0.4683(14)$ & $0.188(3)$ & $2.1(3)$ \\
\hline$O(8)$ & $0.763(4)$ & $0.5746(18)$ & $0.055(4)$ & $3.6(4)$ \\
\hline OW $(1) \dagger$ & $0.013(2)$ & $-0.1158(13)$ & $0.107(2)$ & $1.1(3)$ \\
\hline OW $(2) \dagger$ & $0.058(2)$ & $0.3407(13)$ & $0.130(2)$ & $1.1(2)$ \\
\hline
\end{tabular}

tWater molecule. 
Table 2. Interatomic bond distances $(\AA)$ and angles $($.

Octahedron $\mathrm{Al}(1)$

(2x) $\mathrm{Al}\left(1^{\mathrm{i}}\right)-\mathrm{O}\left(1^{\mathrm{v}}\right): 1.873(14)$

$(2 \times) \mathrm{O}\left(1^{\mathrm{v}}\right)-\mathrm{Al}\left(1^{\mathrm{i}}\right)-\mathrm{OW}\left(1^{\mathrm{i}}\right): 91.1(0.7)$

(2x) $\mathrm{Al}\left(1^{\mathrm{i}}\right)-\mathrm{O}\left(2^{\mathrm{i}}\right): 1.903(13)$

$(2 \times) \mathrm{O}\left(2^{\mathrm{i}}\right)-\mathrm{Al}\left(1^{\mathrm{i}}\right)-\mathrm{OW}\left(1^{\mathrm{i}}\right): 92.2(0.7)$

(2x) $\mathrm{Al}\left(1^{\mathrm{i}}\right)-\mathrm{OW}\left(1^{\mathrm{i}}\right): 1.88(2)$

$(2 \times) \mathrm{O}\left(1^{\mathrm{v}}\right)-\mathrm{Al}\left(1^{\mathrm{i}}\right)-\mathrm{O}\left(2^{\mathrm{i}}\right) \quad: 88.7(0.6)$

Octahedron $\mathrm{Al}(2)$

(2x) $\mathrm{Al}\left(2^{\mathrm{i}}\right)-\mathrm{O}\left(4^{\mathrm{i}}\right): 1.847(15)$

(2x) $\mathrm{Al}\left(2^{\mathrm{i}}\right)-\mathrm{O}\left(7^{\mathrm{i}}\right): 1.91 \quad(2)$

(2x) $\operatorname{Al}\left(2^{\mathrm{i}}\right)-\mathrm{OW}\left(2^{\mathrm{i}}\right): 1.89$ (3)

$\begin{array}{ll}(2 \times) \mathrm{O}\left(4^{\mathrm{i}}\right)-\mathrm{Al}\left(2^{\mathrm{i}}\right)-\mathrm{OW}\left(2^{\mathrm{i}}\right) & : 93.8(0.8) \\ (2 \times) \mathrm{O}\left(4^{\mathrm{i}}\right)-\mathrm{Al}\left(2^{\mathrm{i}}\right)-\mathrm{OW}\left(2^{\mathrm{iv}}\right) & : 83.8(0.8) \\ (2 \times) \mathrm{O}\left(4^{\mathrm{i}}\right)-\mathrm{Al}\left(2^{\mathrm{i}}\right)-\mathrm{O}\left(7^{\mathrm{i}}\right) & : 88.3(0.9) \\ (2 \times) \mathrm{O}\left(7^{\mathrm{i}}\right)-\mathrm{Al}\left(2^{\mathrm{i}}\right)-\mathrm{OW}\left(2^{\mathrm{i}}\right) & : 88.9(0.9) \\ (2 \times) \mathrm{O}\left(7^{\mathrm{i}}\right)-\mathrm{Al}\left(2^{\mathrm{i}}\right)-\mathrm{OW}\left(2^{\mathrm{iv}}\right) & : 93.4(0.9) \\ (1 \times) \mathrm{O}\left(4^{\mathrm{i}}\right)-\mathrm{Al}\left(2^{\mathrm{i}}\right)-\mathrm{O}\left(4^{\mathrm{iv}}\right) & : 88.6(0.8) \\ (1 \times) \mathrm{O}\left(7^{\mathrm{i}}\right)-\mathrm{Al}\left(2^{\mathrm{i}}\right)-\mathrm{O}\left(7^{\mathrm{iv}}\right) & : 95.7(0.9) \\ (2 \times) \mathrm{O}\left(4^{\mathrm{i}}\right)-\mathrm{Al}\left(2^{\mathrm{i}}\right)-\mathrm{O}\left(7^{\mathrm{iv}}\right) & : 171.8(0.9) \\ (1 \times) \mathrm{OW}\left(2^{\mathrm{i}}\right)-\mathrm{Al}\left(2^{\mathrm{i}}\right)-\mathrm{OW}\left(2^{\mathrm{iv}}\right): 176.7(0.8)\end{array}$

Tetrahedron $\operatorname{Cr}(1)$

$(1 \times) \operatorname{Cr}\left(1^{\mathrm{i}}\right)-\mathrm{O}\left(1^{\mathrm{i}}\right): 1.702(12)$

$(1 \times) \mathrm{Cr}\left(1^{\mathrm{i}}\right)-\mathrm{O}\left(2^{\mathrm{i}}\right): 1.645$ (19)

(1x) $\mathrm{O}\left(1^{\mathrm{i}}\right)-\mathrm{Cr}\left(1^{\mathrm{i}}\right)-\mathrm{O}\left(2^{\mathrm{i}}\right): 112.0(0.7)$

(1x) O(1 $\left.1^{\mathrm{i}}\right)-\mathrm{Cr}\left(1^{\mathrm{i}}\right)-\mathrm{O}\left(3^{\mathrm{i}}\right): 109.1(0.9)$

$(1 \times) \mathrm{Cr}\left(1^{\mathrm{i}}\right)-\mathrm{O}\left(3^{\mathrm{i}}\right): 1.57$

$(1 \times) \mathrm{O}\left(1^{\mathrm{i}}\right)-\mathrm{Cr}\left(1^{\mathrm{i}}\right)-\mathrm{O}\left(4^{\mathrm{i}}\right): 108.3(0.8)$

(1×) $\mathrm{Cr}\left(1^{\mathrm{i}}\right)-\mathrm{O}\left(4^{\mathrm{i}}\right): 1.69 \quad(2)$

$(1 \times) \mathrm{O}\left(2^{\mathrm{i}}\right)-\mathrm{Cr}\left(1^{\mathrm{i}}\right)-\mathrm{O}\left(3^{\mathrm{i}}\right): 108.0(0.9)$

$(1 \times) \mathrm{O}\left(2^{\mathrm{i}}\right)-\mathrm{Cr}\left(1^{\mathrm{i}}\right)-\mathrm{O}\left(4^{\mathrm{i}}\right): 110.3(0.8)$

$(1 \times) \mathrm{O}\left(3^{\mathrm{i}}\right)-\mathrm{Cr}\left(1^{\mathrm{i}}\right)-\mathrm{O}\left(4^{\mathrm{i}}\right): 109.0(0.9)$

Double tetrahedron $\mathrm{Cr}(2)$

$(2 \times) \mathrm{Cr}\left(2^{\mathrm{i}}\right)-\mathrm{O}\left(5^{\mathrm{i}}\right): 1.763(10)$

$(2 \times) \mathrm{O}\left(5^{\mathrm{i}}\right)-\mathrm{Cr}\left(2^{\mathrm{i}}\right)-\mathrm{O}\left(6^{\mathrm{i}}\right): 106.5(0.8)$

$(2 \times) \mathrm{Cr}\left(2^{\mathrm{i}}\right)-\mathrm{O}\left(6^{\mathrm{i}}\right): 1.593(19)$

$(2 \times) \mathrm{Cr}\left(2^{\mathrm{i}}\right)-\mathrm{O}\left(7^{\mathrm{i}}\right): 1.608(18)$

(2x) $\mathrm{Cr}\left(2^{\mathrm{i}}\right)-\mathrm{O}\left(8^{\mathrm{i}}\right): 1.64(4)$

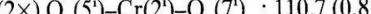

$(2 \times) \mathrm{O}\left(5^{\mathrm{i}}\right)-\mathrm{Cr}\left(2^{\mathrm{i}}\right)-\mathrm{O}\left(8^{\mathrm{i}}\right): 110.6(1.0)$

$(2 \times) \mathrm{O}\left(6^{\mathrm{i}}\right)-\mathrm{Cr}\left(2^{\mathrm{i}}\right)-\mathrm{O}\left(7^{\mathrm{i}}\right): 112.3(1.2)$

(1X) $\operatorname{Cr}\left(2^{\mathrm{i}}\right)-\operatorname{Cr}\left(2^{\mathrm{vii}}\right): 3.179(8)$

(2x) O $\left(6^{\mathrm{i}}\right)-\mathrm{Cr}\left(2^{1}\right)-\mathrm{O}\left(8^{\mathrm{i}}\right): 109.4(1.4)$

(2x) O ( $\left.7^{\mathrm{i}}\right)-\mathrm{Cr}\left(2^{\mathrm{i}}\right)-\mathrm{O}\left(8^{\mathrm{i}}\right): 107.4(1.3)$

$(1 \times) \mathrm{Cr}\left(2^{\mathrm{i}}\right)-\mathrm{O}\left(5^{\mathrm{i}}\right)-\mathrm{Cr}\left(2^{\text {vii }}\right): 128.7(0.4)$

Hydrogen bonds $\mathrm{OW}(1)$

$\mathrm{OW}\left(1^{\mathrm{i}}\right)-\mathrm{O}\left(3^{\mathrm{iv}}\right): 2.81$ (4) (Intra sheet bond)

OW $\left(1^{i}\right)-O\left(6^{\text {viii }}\right): 2.69(2) \quad$ (Inter sheet bond)

$\mathrm{O}\left(3^{\mathrm{iv}}\right)-\mathrm{OW}\left(1^{\mathrm{i}}\right)-\mathrm{O}\left(6^{\text {viii }}\right): 101.5(0.9)$

$\mathrm{Al}\left(1^{\mathrm{i}}\right)-\mathrm{OW}\left(1^{\mathrm{i}}\right)-\mathrm{O}\left(3^{\mathrm{iv}}\right): 121.1(1.0)$

$\mathrm{Al}\left(1^{\mathrm{i}}\right)-\mathrm{OW}\left(1^{\mathrm{i}}\right)-\mathrm{O}\left(6^{\text {viii }}\right): 122.2(1.1)$

Hydrogen bonds OW(2)

$\mathrm{OW}\left(2^{\mathrm{i}}\right)-\mathrm{O}\left(1^{\mathrm{v}}\right): 2.836(18) \quad$ (Intra sheet bond)

$\mathrm{OW}\left(2^{\mathrm{i}}\right)-\mathrm{O}\left(8^{\mathrm{ix}}\right): 2.79 \quad(5) \quad$ (Inter sheet bond)

$\mathrm{O}\left(1^{\mathrm{v}}\right)-\mathrm{OW}\left(2^{\mathrm{i}}\right)-\mathrm{O}\left(8^{\mathrm{ix}}\right): 101.8(0.9)$

$\mathrm{Al}\left(2^{\mathrm{i}}\right)-\mathrm{OW}\left(2^{\mathrm{i}}\right)-\mathrm{O}\left(1^{\mathrm{v}}\right): 119.7(0.9)$

$\mathrm{Al}\left(2^{\mathrm{i}}\right)-\mathrm{OW}\left(2^{\mathrm{i}}\right)-\mathrm{O}\left(8^{\mathrm{ix}}\right): 123.4(1.2)$ 